\title{
Diamond Sensors for Energy Frontier Experiments
}

\author{
Steve Schnetzer \\ for the RD42 Collaboration * \\ Rutgers University \\ E-mail: steves@physics.rutgers.edu
}

\begin{abstract}
We discuss the use of diamond sensors in high-energy, high-intensity collider experiments. Results from diamond sensor based beam conditions monitors in the ATLAS and CMS experiments at the CERN Large Hadron Collider (LHC) are presented and plans for diamond based luminosity monitors for the upcoming LHC run are described. We describe recent measurements on single crystal diamond sensors that indicate a polarization effect that causes a reduction of charge collection efficiency as a function of particle flux. We conclude by describing new developments on the promising technology of 3D diamond sensors.
\end{abstract}

22nd International Workshop on Vertex Detectors

September 15th-20th, 2013

Lake Starnberg, Germany

* See appendix for the full RD42 author list. 


\section{Diamond detector applications in ATLAS and CMS}

Diamond sensors have had wide-spread use as beam conditions monitors in collider detector and other high energy physics experiments. Here we focus on applications in the two multi-purpose detectors CMS and ATLAS at the Large Hadron Collider (LHC) at CERN.

\subsection{Current applications}

Both CMS and ATLAS have employed diamond sensors to monitor the quality of the colliding beams. The primary function is to protect sensitive detector components from possible anomalous beam backgrounds.

The ATLAS Beam Conditions Monitor (BCM) [1] consists of two stations (one on each end of the collision point) each with four detector modules arranged symmetrically in azimuth at a distance of $\pm 184 \mathrm{~cm}$ from the collision point and $5.5 \mathrm{~cm}$ from the beam line. Each module consists of two back-to-back polycrystalline CVD diamond sensors grown by Element Six Ltd. The diamond sensors are each $500 \mu \mathrm{m}$ thick with active areas of $8 \mathrm{~mm} \times 8 \mathrm{~mm}$. They are biased at $2 \mathrm{~V} / \mu \mathrm{m}$ and are readout in parallel by fast electronics with a timing resolution of $0.7 \mathrm{~ns}$ that discriminates beam related background particles from those produced in the proton-proton interactions. The BCM also provides a measurement of bunch-by-bunch luminosities in ATLAS by counting the in-time particles. During the 2011 running the ATLAS BCM provided stable measurements with little or no deviation due to event pileup. It achieved a stable luminosity measurement with a precision of $1.8 \%$.

The CMS Beam Conditions Monitor [2] consists of several systems used to protect the sensitive CMS detector components and to provide beam conditions feedback to LHC operation. Three of these systems are based on diamonds sensors. The BCM1L and BCM2 systems use polycrystalline CVD diamonds sensors readout in current mode with integration times from $40 \mu \mathrm{s}$ to $83 \mathrm{~s}$. In the case of adverse beam conditions, a high current in these sensors initiates a beam abort. The BCM1L is composed of four diamond detectors on each end of CMS at a distance of $\pm 180 \mathrm{~cm}$ from the collision point and $4.5 \mathrm{~cm}$ from the beam line. The BCM2 consists of two detector rings on each end of CMS at a distance of $\pm 14.4 \mathrm{~m}$ from the collision point. The inner rings consist of four diamond detectors at a radius of $5 \mathrm{~cm}$ and the outer rings consist of eight detectors at a radius of $28 \mathrm{~cm}$. All of the diamonds sensors were grown by Element Six Ltd. They are each $10 \mathrm{~mm}$ $\times 10 \mathrm{~mm}$ in area, $400 \mu \mathrm{m}$ thick and are biased at $0.5 \mathrm{~V} / \mu \mathrm{m}$. The Fast Beam Conditions Monitor (BCM1F) [3] is based on single-crystal diamond sensors readout by a high-speed, charge-sensitive, pre-amplifier that provides sensitivity to individual particles. Four modules, consisting of a sensor, a pre-amplifier and an optical driver, are arranged around the beam pipe at a distance of $4.5 \mathrm{~cm}$ from the beam axis at $180 \mathrm{~cm}$ on both sides of the collision point. The single crystal diamond sensors are $5 \mathrm{~mm} \times 5 \mathrm{~mm}$ in area, $500 \mu \mathrm{m}$ thick and are biased at $1.0 \mathrm{~V} / \mu \mathrm{m}$. Figure 1 shows an example of the measurement of the beam profile in the horizontal $(x)$ plane during an LHC Van der Meer scan. Figure 2 shows a comparison of the BCM1F measured beam width compared to those of the CMS pixel detector and forward calorimeter. The agreement among these is within $1.5 \%$ with the BCM1F measurement systematically lower by about $1 \%$. 


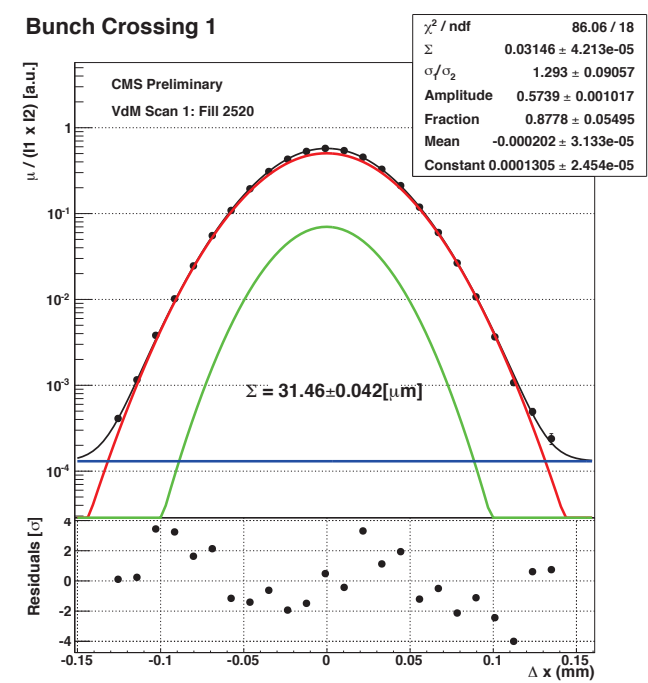

Figure 1: The BCM1F rate normalized to the product of the bunch currents as a function of scan position in the $x$ direction. The black curve is a double Gaussian plus constant fit. The red and green curves are the two Gaussians and the blue curve is the constant baseline.

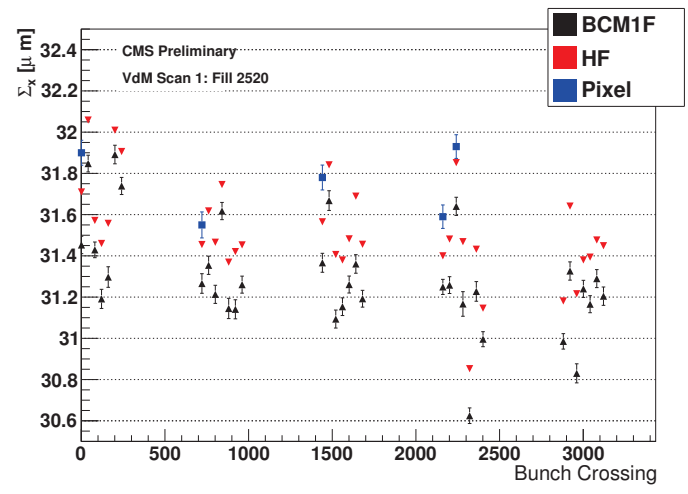

Figure 2: Comparisons of the BCM1F measurements with those of the pixel and forward calorimeter $(\mathrm{HF})$ of the horizontal $(x)$ bunch width during a Van der Meer scan.

\subsection{Future (2015) application}

Both ATLAS and CMS are installing second generation devices, the ATLAS Diamond Beam Monitor (DBM) [ [] [ [5] and the CMS Pixel Luminosity Telescopes (PLT) [6], that will be used to measure the relative bunch-by-bunch luminosity to high precision during the 2015 LHC run. Both of these devices consist of arrays of small-angle, 3-plane telescopes on each end of the collision point. In order to handle the higher rates, both devices will use pixellated sensors for the telescope planes. The ATLAS DBM has four telescopes per end for a total of 24 sensors. The planes are $18 \mathrm{~mm} \times 21 \mathrm{~mm}$ polycrystalline diamonds bump-bonded to the FE-14 [团] ATLAS pixel chip. Most of the polycrystalline CVD diamonds used in the DBM were grown by Element Six Ltd. but about one third were supplied by a relatively new vendor, II-VI Inc. The DBM was installed in the fall of 2013. The CMS PLT has eight telescopes per end for a total of 48 sensors. In the current baseline design of the PLT, the planes are $10 \mathrm{~mm} \times 10 \mathrm{~mm}$ cooled silicon sensors bump bonded to the CMS PSI46V2 [8] pixel readout chip. The PLT will be installed in the fall of 2014.

Both the DBM and PLT will use the number of telescopes with 3-fold coincidences in each bunch crossing $(40 \mathrm{MHz})$ as a measure of the bunch-by-bunch luminosity. Both detectors will also readout the full pixel information at a few $\mathrm{kHz}$. This pixel information will provide a powerful means of reducing systematic errors by determining accidentals and monitoring the plane efficiencies. Both devices are expected to achieve a precision of the order of $1 \%$ on the relative bunch-by-bunch luminosity.

\section{Recent measurements of single-crystal diamond sensors}

Single crystal sensors yield full charge collection at fields between $0.1 \mathrm{~V} / \mu \mathrm{m}$ and $0.4 \mathrm{~V} / \mu \mathrm{m}$ in 
contrast with polycrystalline diamonds sensors that have less that $50 \%$ charge collection even up to fields of $1 \mathrm{~V} / \mu \mathrm{m}$. In addition, the pulse height distribution of single crystal sensors is well separated from pedestal while that of polycrystalline sensors has a low pulse height tail due to spatial nonuniformities. Because of these excellent properties, the original design for the CMS PLT was based on the use of single crystal diamond sensors. Because single crystal CVD diamond must be grown homoepitaxially on diamond substrates produced by a high-pressure, high-temperature process, their size is restricted to less than $5 \mathrm{~mm} \times 5 \mathrm{~mm}$. This small sensor area is not a limitation for the PLT because of the high expected particle rates. Single crystal diamond sensors for the PLT were grown by Element Six are $4.7 \mathrm{~mm} \times 4.7 \mathrm{~mm}$ in area and $500 \mu \mathrm{m}$ thick.

During the 2012-2013 LHC run, a pilot PLT detector was installed in the very forward region of CMS $14.5 \mathrm{~m}$ from the collision point and $5 \mathrm{~cm}$ from the beam line. The pilot detector consisted of four telescopes with single crystal diamond sensors and one telescope with uncooled silicon sensors arranged azimuthally around the beam line. The pilot detector was in place during the entire 2012-2013 run corresponding to an accumulated luminosity of about $20 \mathrm{fb}^{-1}$.

The particle flux in region where the pilot detector was installed, downstream of the forward calorimeter, is more severe than that in the nominal location of the PLT. FLUKA calculations [9] indicate that the charged hadron and neutron fluences integrated over the entire 2012-2013 LHC run of $20 \mathrm{fb}^{-1}$ were $4 \times 10^{13} \mathrm{~cm}^{-2}$ and $5 \times 10^{13} \mathrm{~cm}^{-2}$, respectively. The charged hadrons were predominantly pions with energies greater than $1 \mathrm{GeV}$ while the neutrons had a broad spectrum from $1 \mathrm{KeV}$ to $10 \mathrm{MeV}$ peaking at about $200 \mathrm{KeV}$. Using a Displacement Per Atom (DPA) scoring, these fluences correspond to a net fluence of $3 \times 10^{14} \mathrm{~cm}^{-2}$ of $24 \mathrm{GeV}$ proton equivalents.

Early in the pilot run, a large dependence of the diamond pulse height on particle rate was observed. Figure 3 shows the pulse height of a typical diamond plane at the start of an LHC fill. Before the beams are brought into collision there is a low particle rate of about $100 \mathrm{~Hz}$ in the PLT planes due to beam halo and other beam associated backgrounds. The mean collected charge corresponds to full charge collection. After the beams are brought into collision, the particle rate increases dramatically to about $4 \mathrm{MHz}$. The diamond pulse heights then decrease by $25 \%$ to $20 \%$ over about a 10 second period.

This decrease in diamond pulse height with rate becomes larger with increasing integrated fluence. Figure $⿴$ shows the pulse height distributions for a typical diamond sensor for particle rates of $30 \mathrm{kHz}$ and $4 \mathrm{MHz}$ for integrated fluences of $6 \times 10^{12} \mathrm{~cm}^{-2}$ and $1 \times 10^{14} \mathrm{~cm}^{-2}$ of $24 \mathrm{GeV}$ proton equivalents. The $30 \mathrm{kHz}$ data was taken during the reduced intensity in a Van der Meer scan while the $4 \mathrm{MHz}$ data is during normal beam collisions. There is a decrease of pulse height with increased fluence. At the highest fluence of $1 \times 10^{14} \mathrm{~cm}^{-2}$ of $24 \mathrm{GeV}$ proton equivalents, the pulse height is highly reduced even at lower particle rate. The pulse heights were measured with the diamond pixel sensor. Each pixel had a threshold of between 3,000 and 3,500 electrons. This provides a lower limit on the measured pulse height since a signal lower than the threshold would not trigger a pixel readout. The pulse height distributions for the higher fluence are mostly single pixel cluster hits that are near to the pixel threshold. A rate dependence at the higher fluence is still seen but it is reduced because of the lower overall pulse height.

Also shown in Fig. 4 , is the pulse height distribution of an unirradiated single crystal diamond at particle rates of $10 \mathrm{kHz}$ and $2 \mathrm{MHz}$ that was measured in a $250 \mathrm{MeV} / \mathrm{c}$ pion beam at the PSI cyclotron. This unirradiated diamond has full charge collection at both rates and shows no rate 


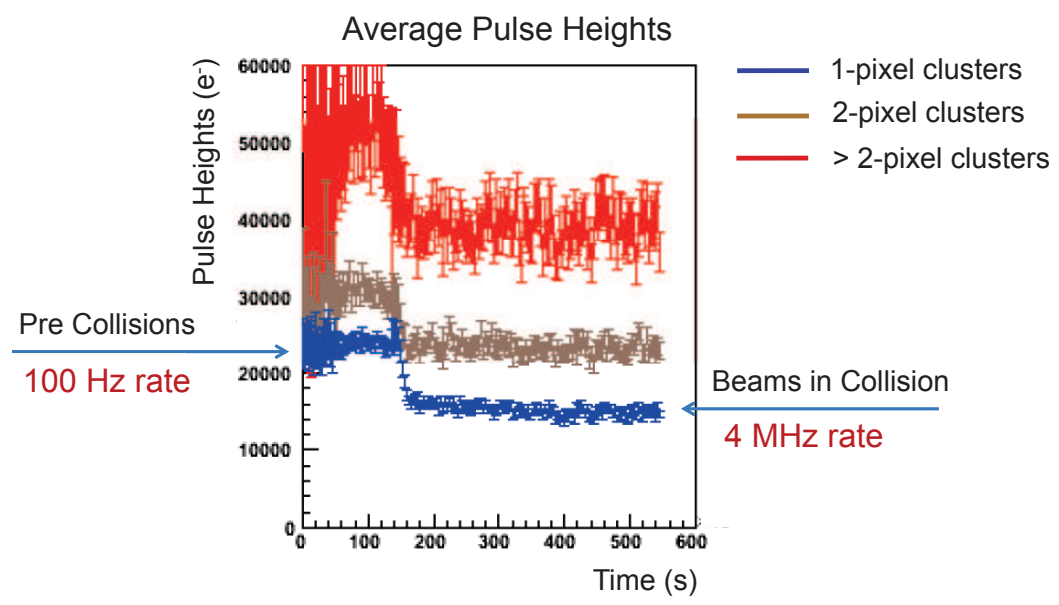

Figure 3: Time dependence of diamond sensor pulse height at start of LHC fill when particle flux changes from low pre-collision rate to high rate during collisions. Blue, brown and red data are for single-pixel, two-pixel and more than two-pixel cluster hits respectively.

dependence. Diamonds that were used in the pilot run were also tested in the PSI beam. These showed a decrease in pulse height and a dependence on rate consistent with that observed during the pilot run.

A possible explanation for the observed effect is build-up of trapped space charge that produces a polarization field in the diamond opposing the applied charge collection field. Traversing ionizing particles produce electrons and holes uniformly throughout diamond. Due to the applied field, electrons drift toward one electrode and holes to the other. As a result, there is higher current density of electrons (holes) on the positive (negative) voltage side of the diamond. The presence of long-lived traps in the diamond causes negative (positive) space charge to build up on the positive (negative) voltage side of the diamond producing an electric field in the direction opposing the applied field. This results in a reduced net electric field that leads to lower drift velocity and longer collection times for the electrons and holes, thereby increasing the trapping probability and reducing the charge collection efficiency. The number of space charge traps increases with increasing fluence, thereby, leading to an enhanced effect. This model predicts that the effect would be reduced by increasing the applied field so that the induced opposing polarization field would have less effect. This was observed during the pilot run. At the lower fluences, the pulse could be recovered and the rate effect reduced by doubling the applied field to $1 \mathrm{~V} / \mu \mathrm{m}$.

If this model is correct, an important question is whether the space charge traps responsible for the polarization are predominantly at the diamond surfaces or are distributed throughout the bulk. In order to test this, material was removed from both surfaces of the single crystal diamond by Reactive Ion Etching (RIE). RIE provides a means of removing diamond material without causing the lattice damage that can occur during mechanical removal. Several of the diamond sensors that were in the pilot run had three microns of material removed from both surfaces by RIE. These were then tested in the pion beam at the PSI cyclotron. The RIE processing gave no measurable improvement in the charge collection efficiency or reduction in the rate dependence. A total of twelve microns of material was then removed from both surfaces of several pilot run diamonds. In 

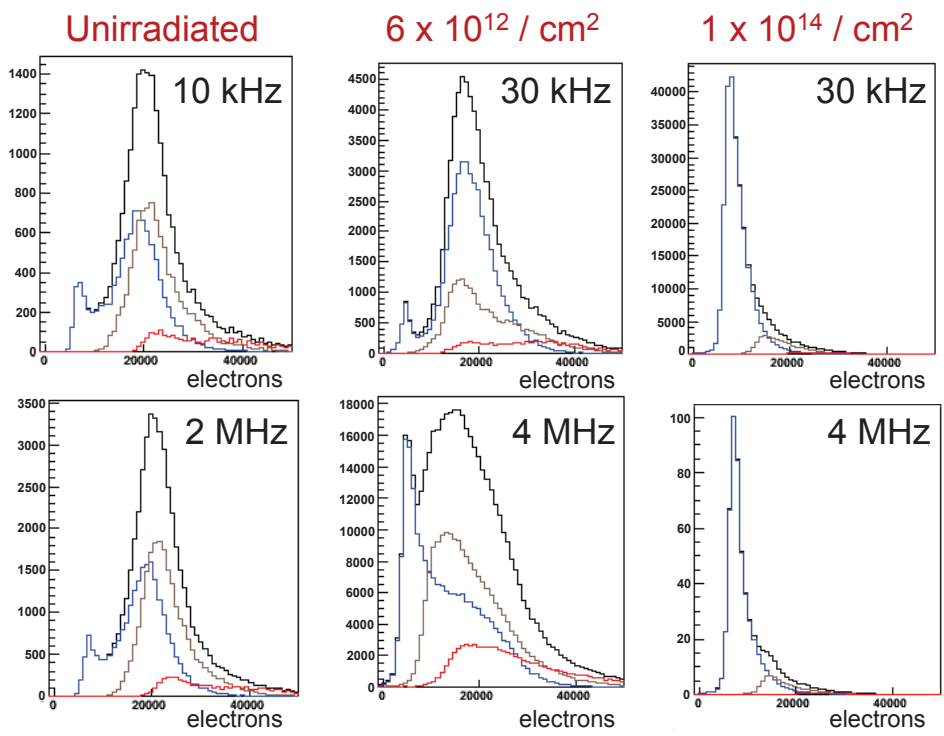

Figure 4: Low and high rate pulse height distributions for an unirradiated sensor and for sensors that received fluences of $6 \times 10^{12}$ and $1 \times 10^{14}$ of $24 \mathrm{GeV}$ proton equivalent per $\mathrm{cm}^{2}$ during the PLT pilot run. The color coding of the number of pixels in the hit cluster is same as in Fig. 3.

bench measurement with a ${ }^{90} \mathrm{Sr}$ beta source no improvement in charge collection was seen. The conclusion from these studies is that the polarization traps are in the bulk and not concentrated near the surface. It was, however, found that RIE processing improved the current/voltage characteristics of the diamond sensors. Following RIE processing, all samples were able to hold a field of $1 \mathrm{~V} / \mu \mathrm{m}$ with negligible leakage current $(<1 \mathrm{pA})$. Removal of approximately 1 micron of material from each surface is recommended as part of the preparation of the surface for metallization.

A study is currently underway to determine whether these effects are also present in polycrystalline diamond and whether it is proton or neutron irradiation that is primarily responsible. Samples of both polycrystalline and signal crystal diamond will be irradiated separately with neutrons and protons. Single electrode and pixel electrode detectors fabricated from these samples will then be tested in a high rate beam at PSI. Results are expected by June of 2014.

\section{3D diamond sensors}

As for silicon sensors, the radiation hardness of diamond sensors can potentially be significantly enhanced by collecting the charge on 3D column electrodes rather than on conventional surface electrodes [10] [11]. The 3D column electrode structure allows the drift distance to be comparable to the carrier mean free path leading to less trapping of the drifting charge. An advantage of 3D diamond sensors over silicon is that in principle the column electrodes can be fabricated 
more simply by laser processing than by the multi-step wafer processing required to form column electrodes in silicon [12]. In this process, a femto-second laser with traveling-focus is used to produce a column of conductive material with resistivity of the order of $1 \Omega \cdot \mathrm{cm}$. We discuss here initial beam studies of a prototype diamond 3D sensor.

In Fig. 5 we show a photograph of the first 3D diamond detector fabricated on a $500 \mu \mathrm{m}$ thick single crystal diamond sensor. For this device, the 3D cells in a row were connected by strips which could be read out easily without an individual connection to each cell. The strips were made with a Chromium-Gold contact. Alternating rows were connected to a common bias while intervening signal rows were wire-bonded to a strip VA readout chip[13] with $150 \mu \mathrm{m}$ pitch as shown in Figure 5. With $150 \mu \mathrm{m}$ spacing between signal columns, the drift length between bias and signal column is $106 \mu \mathrm{m}$. The yield of working columns was approximately $90 \%$.

A pattern consisting of three regions shown in Figure 6 was fabricated on the prototype 3D sensor: a region of strip electrodes with a back side contact, a region of the 3D surface pattern but with no columns and a region of 3D surface pattern with columns. The purpose of the no column region was to determine the size of the signal induced due to the surface metallization alone.
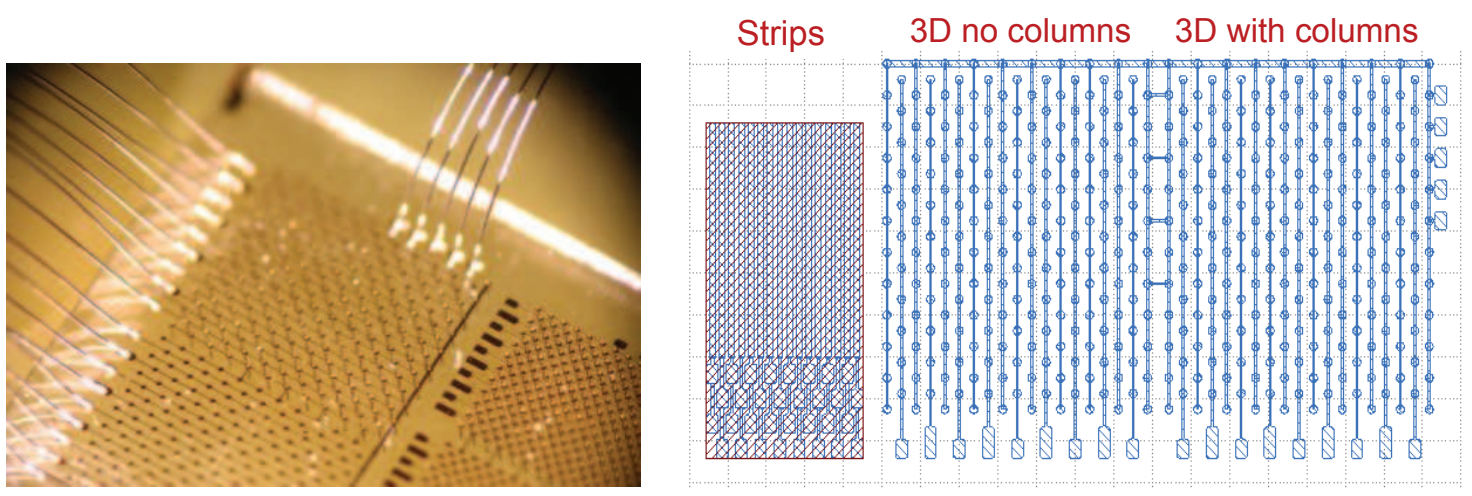

Figure 5: Picture of the surface metallization of the $3 \mathrm{D}$ sensor.

Figure 6: Surface metallization pattern on the 3D diamond prototype.

This prototype 3D detector was tested in a $120 \mathrm{GeV}$ proton beam at the CERN SPS. An eight plane silicon strip tracking telescope (the Strasbourg Telescope)[14] was used to define the location of particle tracks on the 3D sensor with a position resolution of the order of a few microns. A diamond strip detector fabricated on the same $500 \mu \mathrm{m}$ single crystal diamond with $50 \mu \mathrm{m}$ pitch strips operating at $500 \mathrm{~V}$ collected data simultaneously with the 3D detector and was used to calibrate the charge collection. The strip detector fully collected the charge at this operating voltage.

After appropriately choosing a fiducial region, the two-strip clustered pulse height of the diamond strip detector operating at $500 \mathrm{~V}$ and the diamond 3D detector operating at $25 \mathrm{~V}$ were compared. Figure 7 shows the pulse height comparisons of the two detectors for tracks within the fiducial region of each detector. Both distributions have a most probable value corresponding to full charge collection and are very similar. The pulse height distribution for the strip electrode readout is described well by a Landau function while the 3D electrode readout has slight deviations from this shape which are being investigated.

A first proof-of-principle of a 3D diamond sensor has been established with sensor achieving 


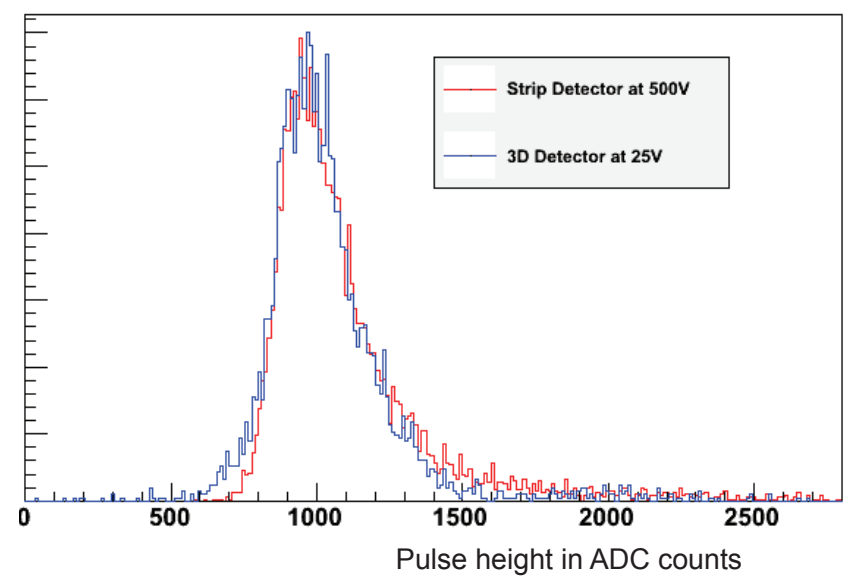

Figure 7: Pulse height distribution for strip readout (red) and 3D column readout (blue) for those tracks in the fiducial region of each detector.

a signal response comparable to that for a strip detector. The next steps will be to test the performance of 3D diamond sensors after irradiation and to fabricate and test polycrystalline 3D diamond sensors. In addition, work is ongoing to improve the quality of 3D detectors produced and to try alternative production approaches.

\section{Summary}

Diamond sensors have seen wide spread application in high energy physics and have been successfully implemented in the beam monitoring systems of the ATLAS and CMS detectors. Second generation beam monitors based on diamond pixel sensors that will measure the bunch-by-bunch luminosity to high precession will be installed for the 2015 LHC run. A problem regrading rate dependence of the pulse height of single crystal diamond sensors was observed in a pilot run of the CMS PLT. This dependence increases with increasing irradiation and is attributed to polarization due to build-up of space charge. An effort is in progress to further investigate this effect and to determine if it is also present in polycrystalline diamond. The promising technology of 3D diamond sensors is being developed. A prototype 3D sensor based on a single crystal diamond has been successfully fabricated and tested. Future developments will include an investigation of the radiation tolerance of 3D diamond sensors and fabrication and testing of polycrystalline 3D diamonds sensors.

\section{Acknowledgments}

The RD42 Collaboration would like to gratefully acknowledge the staff of the PRISM micro fabrication laboratory at Princeton University and in particular Bert Harrop for their excellent and dedicated efforts in the metallization and bump-bonding of diamond pixel detectors and for their rapid development of Reactive Ion Etching processing of diamonds. We would also like to thank the beam line staffs at the CERN SPS and the Paul Scherrer 
Institute (PSI) Cyclotron for their invaluable assistance in carrying out the diamond detector tests. We would like to especially thank Konrad Deiters and Davide Reggiani of PSI for their expert and invaluable assistance.

\section{References}

[1] V. Cindro et al., The ATLAS Beam Conditions Monitor, JINST 3 (2008) P02004 .

[2] A. E. Dabrowski et al., The performance of the Beam Conditions and Radiation Monitoring System of CMS, IEEE Nucl.Sci.Symp.Conf.Rec. (2011) 489-495.

[3] R. S. Schmidt et al., Performance of the Fast Beam Conditions Monitor BCMIF of CMS in the first running periods of LHC, JINST 6 (2011) C01004.

[4] M. Capeans, et al., ATLAS Insertable B-Layer Technical Design Report CERN/LHCC-2010-013; ATLAS-TDR-19, Sep 2010.

[5] M. Cerv, The ATLAS Diamond Beam Monitor, submitted to JINST 9 (2014) C02026.

[6] E. Halkiadakis et al., A proposed luminosity monitor for CMS based on small angle diamond pixel telescopes, Nucl.Instrum.Meth. A565 (2006) 284-289.

[7] M. Barbero et al., FE-14 ATLAS pixel chip design, PoS Vertex 2009 Proceedings 027.

[8] H.Chr. Kastli et al., Design and performance of the CMS pixel detector readout chip, Nucl.Instrum.Meth. $\mathbf{A 5 6 5}$ (2006) 188-194.

[9] M. Guthoff et al., Radiation damage in the diamond based beam condition monitors of the CMS experiment at the Large Hadron Collider (LHC) at CERN, Nucl.Instrum.Meth. A730 (2013) 168-173.

[10] C. Da Via, J. Hasi, C. Kenney, A. Kok and S. Parker, 3D silicon detectors status and applications, Nucl.Instrum.Meth. A549 (2005) 122-125.

[11] C. Da Via et al., Future trends of 3D silicon sensors, Nucl.Instrum.Meth. A731 (2013) 201-204.

[12] A. Oh, B. Caylar, M. Pomorski and T. Wengler, A novel detector with graphitic electrodes in CVD diamond, Diamond \& Related Materials 38 (2013) 9-13.

[13] Integrated Detector and Electronics (IDEAS). The VA Circuits, www.ideas.no., Pb.315, Veritasveien 9, N-1322 Hovik, Norway.

[14] W. Adam et al., A CVD diamond beam telescope for charged particle tracking, IEEE Trans.Nucl.Sci. 49 (2002) 1857-1862.

\section{A. The RD42 author list}

M. Cristinziani, ${ }^{a}$ L. Gonella, ${ }^{a}$ F. Hügging, ${ }^{a}$ J. Janssen, ${ }^{a}$ T. Obermann, ${ }^{a}$ N. Wermes, ${ }^{a}$ V. Bellini, ${ }^{b}$ S. Costa, ${ }^{b}$ R. Potenza, ${ }^{b}$ C. Sutera, ${ }^{b}$ C. Tuve, ${ }^{b}$ M. Bartosik, ${ }^{c}$ M. Cerv, ${ }^{c}$ A. Dabrowski, ${ }^{c}$ B. Dehning, ${ }^{c}$ D. Dobos,${ }^{c}$ C. Gallrapp,${ }^{c}$ M. Gastal, ${ }^{c}$ H. Jansen,${ }^{c}$ C. Kurfuerst, ${ }^{c}$ H. Pernegger ${ }^{c}$ S. Roe, ${ }^{c}$ A. Sfyrla, ${ }^{c}$ P. Weilhammer, ${ }^{c}$ C. Weiss, ${ }^{c}$ T. Wengler, ${ }^{c}$ H. FraisKölbl, ${ }^{d}$ T. Schreiner, ${ }^{d}$ M. Bruzzi, ${ }^{e}$ R. D’Alessandro, ${ }^{f}$ S. Lagomarsino, ${ }^{f}$ G. Parrini, ${ }^{f}$ S. Sciortino, ${ }^{f}$ S. Kwan, ${ }^{g}$ L. Uplegger, ${ }^{g}$ E. Berdermann, ${ }^{h}$ M. Kis, ${ }^{h}$ M. Traeger,${ }^{h}$ V. Eremin, ${ }^{i}$ J-M. Brom, ${ }^{j}$ G. Claus,${ }^{j}$ W. Dulinski,${ }^{j}$ M. Goffe,${ }^{j}$ J. Hosslet,${ }^{j}$ C. Maazouzi, ${ }^{j}$ C. Mathieu, ${ }^{j}$ 
A. Golubev, ${ }^{k}$ E. Grigoriev, ${ }^{k}$ S. Kuleshov, ${ }^{k}$ G. Shimchuk, ${ }^{k}$ V. Cindro, ${ }^{l}$ A. Gorišek, ${ }^{l}$ G. Kramberger, ${ }^{l}$ I. Mandic, ${ }^{l}$ M. Mikuž, ${ }^{l}$ M. Zavrtanik, ${ }^{l}$ W. de Boer, ${ }^{m}$ M. Guthoff, ${ }^{m}$ P. Bergonzo, ${ }^{n}$ B. Caylar, ${ }^{n}$ M. Pomorski,${ }^{n}$ D. Tromson, ${ }^{n}$ V. Belyaev, ${ }^{o}$ K.K. Gan, ${ }^{q}$ H. Kagan, ${ }^{q}$ R. Kass, ${ }^{q}$ H. Merritt, ${ }^{q}$ J. Moss, ${ }^{q}$ S. Phan, ${ }^{q}$ S. Smith,,${ }^{q}$ D. Hidas, ${ }^{r}$ S. Schnetzer, ${ }^{r}$ R. Stone, ${ }^{r}$ J. Forneris ${ }^{s}{ }^{s}$ A. Lo Giudice,${ }^{s}$ P. Olivero ${ }^{s}{ }^{s}$ F. Picollo, ${ }^{s}$ A. Re ${ }^{s}$ E. Vittone ${ }^{s}$ C. Chau, ${ }^{t}$ G. McGoldrick, ${ }^{t}$ N. Venturi, ${ }^{t}$ W. Trischuk, ${ }^{t}$ M. Fisher-Levine, ${ }^{w}$ J. Goldstein, ${ }^{w}$ J. Velthuis, ${ }^{w}$ G. Oakham, ${ }^{x}$ D. Chren, ${ }^{y}$ B. Sopko, ${ }^{y}$ J. Cumalat, ${ }^{z}$ K. Stenson, ${ }^{z}$ S. Wagner, ${ }^{z}$ M. Artuso, ${ }^{a a}$ A. Borgia, ${ }^{a}{ }^{a}$ J. Garofoli, ${ }^{a}{ }^{a}$ R. Mountain, ${ }^{a}{ }^{a}$ J.C. Wang, ${ }^{a}$ M. Hoeferkamp, ${ }^{b b}$ S. Seidel, ${ }^{b b}$ R. Wang, ${ }^{b b}$ I. Haughton, ${ }^{c c}$ L. Lin, ${ }^{c c}$ A. Oh, ${ }^{c c}$ V. Tyzhnevyi, ${ }^{c c}$ D. Whitehead, ${ }^{c c}$ L. Graber, ${ }^{d d}$ J. Grosse-Knetter, ${ }^{d d}$ A. Quadt, ${ }^{d d}$ J. Weingarten, ${ }^{d d}$ F. Bachmair, ${ }^{e e}$ L. Bäni, ${ }^{e e}$ D. Hits, ${ }^{e e}$ R. Wallny, ${ }^{e e}$ R. Eusebi, ${ }^{f f}$ S. Spanier, ${ }^{g g}$ G. Chiodini, ${ }^{\text {hh }}$ R. Perrino, ${ }^{\text {hh }}$ S. Spagnolo, ${ }^{\text {h }}$

${ }^{a}$ Universität Bonn, Bonn, Germany, ${ }^{b} N F N /$ University of Catania, Catania, Italy, ${ }^{c} C E R N$, Geneva, Switzerland, ${ }^{d}$ FWT, Wiener Neustadt, Austria, ${ }^{e}$ INFN/University of Florence, Florence, Italy, ${ }^{f}$ Department of Energetics/INFN, Florence, Italy, ${ }^{g}$ FNAL, Batavia, USA, ${ }^{h}$ GSI, Darmstadt, Germany, ${ }^{i}$ Ioffe In-

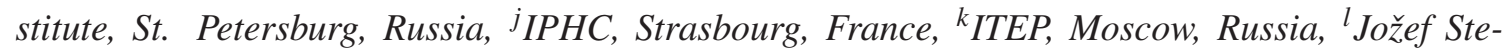
fan Institute, Ljubljana, Slovenia, ${ }^{m}$ Universität Karlsruhe, Karlsruhe, Germany, ${ }^{n}$ CEA-LIST Technologies Avancees, Saclay, France, ${ }^{\circ}$ MEPHI Institute, Moscow, Russia, ${ }^{q}$ The Ohio State University, Columbus, OH, USA, ${ }^{r}$ Rutgers University, Piscataway, NJ, USA, ${ }^{s}$ University of Torino and INFN-Torino, Torino, Italy, ${ }^{t}$ University of Toronto, Toronto, ON, Canada, ${ }^{w}$ University of Bristol, Bristol, UK, ${ }^{x}$ Carleton University, Ottawa, Canada, ${ }^{y}$ Czech Technical Univ., Prague, Czech Republic, ${ }^{z}$ University of Colorado, Boulder, CO, USA, ${ }^{a}$ Syracuse University, Syracuse, NY, USA, ${ }^{b b}$ University of New Mexico, Albuquerque, NM, USA, ${ }^{c c}$ University of Manchester, Manchester, $U K,{ }^{d d}$ Universität Goettingen, Goettingen, Germany, ${ }^{e e}$ ETH Zurich, Zurich, Switzerland, ${ }^{f f}$ Texas A\&M, College Park Station, TX, USA, ${ }^{g g}$ University of Tennessee, Knoxville, TN, USA, ${ }^{\text {hh }}$ INFNLecce, Lecce, Italy 